\title{
Preliminary Modeling of Transfer RNA Kinetics in the Cytoplasm of Escherichia coli Bacteria
}

\author{
Sándor J. Piros ${ }^{1}$ and Ghaleb A. Husseini ${ }^{2, *}$ \\ ${ }^{1}$ Mechatronics Engineering, American University of Sharjah, Sharjah, United Arab Emirates \\ ${ }^{2}$ Department of Chemical Engineering, American University of Sharjah, Sharjah, United Arab Emirates
}

\begin{abstract}
Transfer RNAs (tRNAs) can recognize a specific amino acid from a possible pool of 20 . They are able to transport these protein building-blocks to the ribosome, the site where amino acids assemble into protein chains. Accurate and rapid selection of tRNAs by the ribosome is critical for cell survival. The aim of this paper is to develop a preliminary and simple model of tRNA molecular movement in the bacterial (Escherichia coli-E. coli) cytoplasm. We examine the spatial movement/placement of aminoacylated tRNAs in the cytoplasm-viewed from the perspective of that particular aminoacylated tRNA. To achieve this goal, a kinetic model of the interaction between messenger RNA, ribosome, and RNA molecules is developed. The purpose of the simulation is to examine the conditions necessary for the tRNA to deliver a particular amino acid to the ribosome within a biological timeframe. Simulation results show that it is unlikely that tRNAs are able to reach the "A site" of the ribosome by random movement.
\end{abstract}

\section{INTRODUCTION}

Proteins are the building-block macromolecules of cells. Proteins are assembled from 20 different amino acids; usually a few hundred of them are connected into a chain by peptide bonds. The amino acid sequence of the protein is coded by the messenger RNA (mRNA) and the factory where the assembling takes place is the ribosome. The ribosome is a conglomerate of RNA molecules and proteins. The template of these RNA molecules is stored inside the chromosome in the form of double helix DNA chains containing the genetic information used in the development of all living organisms., ${ }^{1,2}$

The manufacturing of proteins in the ribosome is carried out by the help of tiny robots: the transfer RNA (tRNA) molecules. They are possibly the smallest autonomous robots on earth. They can recognize a specific amino acid from the possible pool of 20 different amino acids. They are able to transport these blocks to the ribosome - the site where amino acids are assembled into a protein chain. A tRNA molecule acts similar to a mobile robot, as it delivers the spare parts (amino acids) to the assembly-line (the ribosome). Extensive research on tRNA interaction with the ribosome exists, but these studies focus on their biochemical interactions. There are other factors of great significance that play an integral part in this assembly. The ribosome is capable of distinguishing the cognate tRNA (ctRNA) from all other tRNA molecules, but it accomplishes this task by trial and error,

*Author to whom correspondence should be addressed. which is extremely time-consuming. It is highly probable that a mechanism exists in nature whereby the correct ctRNA is chosen before it enters the ribosome "A site." It is important to note here that no description on the tRNA movement in the cytoplasm is found in literature, but extensive research is available on tRNA recognition, selection and relative movements into or inside the ribosome. ${ }^{3,4}$

The aim of this paper is to develop a model of tRNA molecular movement in the bacterial cytoplasm and run simulations according to different tRNA concentrations and velocity conditions. The main criterion required in protein synthesis is the availability of the necessary amino acid in the vicinity of the ribosome. Therefore, we examine the spatial movement or placement of aminoacyl-tRNA (aa-tRNA) molecules in the cytoplasm-viewed from the perspective of that particular aa-tRNA.

The significance of this research is that it attempts to present a basic dynamic model to understand tRNA's "modus operandi" in assembling amino acids into a protein chain. The more we know about such a process, the more efficient medications (including antibiotics) we are capable of synthesizing.

\section{THE USE OF ESCHERICHIA COLI (E. COLI) AS A MODEL ORGANISM}

Since protein biosynthesis is similar in prokaryotes and eukaryotes, Escherichia coli (E. coli), a prokaryotic microorganism, has been chosen as a model organism in this study. E. coli is frequently studied in micro- and molecular biology. It is a 
prokaryotic organism with a simple structure that renders it an excellent target for investigation and experimentation. E. coli can also be grown easily and its genetic components are comparatively simple and easy to manipulate, making it one of the most studied prokaryotic model organisms in biotechnology.

The DNA sequence of $E$. coli reveals 4441 open reading frames corresponding to 4322 proteins and 122 ribosomal RNAs (rRNAs) and tRNAs. The entire sequence of the E. coli genome has already been identified. Data and statistics are readily available for the calculations conducted in this research. ${ }^{5,6}$ The model organism E. coli is 2-4 $\mu \mathrm{m}$ long with an average diameter of $500-800 \mathrm{~nm}$. The cytoplasm of the whole cell thus contains, in addition to water molecules, about 40 million molecules or, if we omit ions and small organic molecules, about half a million macromolecules. ${ }^{5}$

Little is known about the movement of macromolecules in bacterial cells. In early experiments, it has been shown that the motion of macromolecules was consistent with simple diffusion on a time scale of $<1 \mathrm{sec}$ and bacterium length of $\sim 1 \mu \mathrm{m} .^{7}$ Deich et al. ${ }^{8}$ investigated individual fluorescently labeled proteins in the membrane of $C$. crescentus and characterized their twodimensional motion as diffusive. Golding et al. have found that the bacterial cytoplasmic motion is subdiffusive on a time scale of seconds to minutes. ${ }^{9}$

\section{METHOD}

Many approaches exist for the simulation of biochemical cellular processes using deterministic and stochastic modeling approaches. Three types of cell models are generally discussed: macroscopic, mesoscopic and microscopic models. ${ }^{5}$ Macroscopic models deal with molecular concentrations determined by stochastic differential equations. Forces between or inside the molecules are ignored. Usually an infinite reaction volume is assumed. Mesoscopic models deal with individual molecular dynamics of biochemical reactions. Generally physical forces between or inside the molecules are not considered. On the other hand, microscopic models are the only models that deal with physical forces within or between molecules. This type of modeling is fine-graded and is not suitable for whole-cell simulation because of computational restrictions, and the nature of the interactions considered. A microscopic model was used for describing molecular folding - e.g., secondary structure of proteins. ${ }^{10,11}$

The relatively small number of tRNAs compared to the number of ribosomes per bacterial cells is another important fact. According to literature, there are approximately ten times more tRNAs present in E. coli than the number of ribosomes. ${ }^{6}$ If the quantity of each amino acid specific tRNA is about $2 \%$, then there is only one tRNA molecule for five ribosomes. Therefore tRNAs are quite busy and well utilized in providing amino acid molecules to the ribosomes.

Most cell simulations concentrate on biochemical interactions, but in this paper we extend our research into the physical domain (to account for physical interactions). The model sought does not fit into any of the above mentioned model categories. Instead of two dimensions, a 3-dimensional model can be used. Particles are considered to follow the rules of Brownian motion. The speed of differently sized particles can be approximated using literature data. The statistical average time interval (i.e., the delivery time of a particular amino acid to the ribosome) when the same tRNA interacts with the same ribosome, is reported in this work. ${ }^{3,12,13}$
Before we discuss our mathematical model, it is important to mentioned that most of the data used in our simulations were taken from Project CyberCell E. coli Statistics. ${ }^{6}$ The average rate of amino acid assembly is around $20 \mathrm{~ms}$ per base, so if our simulation results show a rate of assembly that fits into this time frame, it is possible to conclude that the selection process is purely statistical or random. ${ }^{7}$

\section{MODEL}

\subsection{Particle Collision}

In our proposed model, molecular particles move in 3-dimensional space and collide with each other. The most important part of the simulation program is to detect which particle collides with which object, when and where they collide, as well as be able to calculate each particle's velocity and resulting direction after the collision.

First we need to decide whether collisions between atomic particles are elastic or inelastic. Since molecules could be considered as rigid bodies and their collision energy does not cause any chemical interaction, collisions were considered fully elastic. Available literature in this regards provides descriptions for 1D and $2 \mathrm{D}$ cases. $^{14}$

\subsection{Elastic Collision of Particles}

In elastic collisions, kinetic energy and momentum are conserved, i.e., there is no energy loss in the form of heat, etc.

Kinetic energy (KE) of a moving object is:

$$
\mathbf{E}_{\mathbf{k}}=\frac{1}{2} \mathbf{m} \mathbf{v}^{2}
$$

And its momentum $(\mathbf{P})$ is:

$$
\overrightarrow{\mathbf{P}}=\mathbf{m} \overrightarrow{\mathbf{v}}
$$

Where $\mathbf{m}$ is the mass and $\mathbf{v}$ is the velocity. Velocity and momentum are vectorial quantities.

If we have two objects colliding centrally, then

$$
\sum_{\mathbf{i}} \mathbf{E}_{\mathbf{k}_{\mathbf{i}}}=\sum_{\mathbf{i}} \mathbf{E}_{\mathbf{k}_{\mathbf{i}}}^{\prime}
$$

(the prime indicates the value after collision), and

$$
\sum_{\mathbf{i}} \mathbf{P}_{\mathbf{i}}=\sum_{\mathbf{i}} \mathbf{P}_{\mathbf{i}}^{\prime}
$$

Conservation of kinetic energy and momentum gives Eqs. (5) and (6):

$$
\begin{aligned}
\frac{1}{2} \mathbf{m}_{\mathbf{1}} \mathbf{v}_{\mathbf{1}}^{\mathbf{2}}+\frac{1}{2} \mathbf{m}_{\mathbf{2}} \mathbf{v}_{\mathbf{2}}^{\mathbf{2}}=\frac{1}{2} \mathbf{m}_{\mathbf{1}} \mathbf{v}_{\mathbf{1}}^{\prime \mathbf{2}}+\frac{1}{2} \mathbf{m}_{\mathbf{2}} \mathbf{v}_{\mathbf{2}}^{\prime \mathbf{2}} \\
\mathbf{m}_{\mathbf{1}} \overrightarrow{\mathbf{v}}_{\mathbf{1}}+\mathbf{m}_{\mathbf{2}} \overrightarrow{\mathbf{v}}_{\mathbf{2}}=\mathbf{m}_{\mathbf{1}} \overrightarrow{\mathbf{v}_{\mathbf{1}}^{\prime}}+\mathbf{m}_{\mathbf{2}} \overrightarrow{\mathbf{v}_{\mathbf{2}}^{\prime}}
\end{aligned}
$$

\subsubsection{Elastic Collision in 1 Dimension (1D)}

Object $_{1}$ and Object $_{2}$ are moving at $\mathbf{v}_{\mathbf{1}}$ and $\mathbf{v}_{\mathbf{2}}$ velocities respectively, as shown in Figure 1.

From Eqs. (5) and (6) we are able to calculate the objects velocities after the collision (shown in Fig. 2) as

$$
\mathbf{v}_{1}^{\prime}=\frac{\left(\mathbf{m}_{1}-\mathbf{m}_{2}\right) \mathbf{v}_{1}+2 \mathbf{m}_{2} \mathbf{v}_{2}}{\mathbf{m}_{1}+\mathbf{m}_{2}}
$$

and

$$
\mathbf{v}_{2}^{\prime}=\frac{\left(\mathbf{m}_{2}-\mathbf{m}_{1}\right) \mathbf{v}_{2}+2 \mathbf{m}_{1} \mathbf{v}_{1}}{\mathbf{m}_{1}+\mathbf{m}_{2}}
$$

respectively. ${ }^{14}$ 


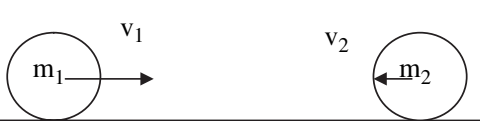

Fig. 1. The figure shows two particles of masses, $\mathbf{m}_{\mathbf{1}}$ underline and $\mathbf{m}_{\mathbf{2}}$, and velocities, $\mathbf{v}_{\mathbf{1}}$ and $\mathbf{v}_{\mathbf{2}}$, before they collide (1-D collision).

\subsubsection{Collision in 2 Dimensions (2D)}

The unit normal vector of a collision is:

$$
\overrightarrow{\mathbf{u}}_{\mathbf{n}}=\frac{\overrightarrow{\mathbf{n}}}{|\overrightarrow{\mathbf{n}}|}
$$

where,

$$
\overrightarrow{\mathbf{n}}=\left(\left(\mathbf{x}_{2}-\mathbf{x}_{1}\right),\left(\mathbf{y}_{2}-\mathbf{y}_{1}\right)\right)
$$

and the unit tangential vector is:

$$
\overrightarrow{\mathbf{u}_{\mathbf{t}}}=\left(-\mathbf{u}_{\mathbf{n}_{\mathbf{y}^{\prime}}} \mathbf{u}_{\mathbf{n}_{\mathbf{x}}}\right)
$$

where, $\left(\mathbf{x}_{1}, \mathbf{y}_{1}\right)$ and $\left(\mathbf{x}_{2}, \mathbf{y}_{2}\right)$ are the coordinates of the objects' centers of mass at the moment of collision $\left(\mathbf{u}_{\mathbf{n}}\right.$ and $\mathbf{u}_{\mathbf{t}}$ are shown in Figs. 3, 4 and 5).

After the collision, tangential velocity components $L$ are unchanged. $\mathbf{v}_{\mathbf{1}_{\mathbf{n}}}$ is the scalar velocity in the normal direction, and $\mathbf{v}_{\mathbf{1}_{\mathbf{t}}}$ is the tangential component.

Using dot products, we calculate the velocity components as

$$
\begin{aligned}
& \mathbf{v}_{\mathbf{1}_{\mathbf{n}}}=\overrightarrow{\mathbf{u}_{\mathrm{n}}} \cdot \overrightarrow{\mathbf{v}_{1}} \\
& \mathbf{v}_{\mathbf{1}_{\mathrm{t}}}=\overrightarrow{\mathbf{u}_{\mathrm{t}}} \cdot \overrightarrow{\mathbf{v}_{\mathbf{1}}}
\end{aligned}
$$

and

$$
\begin{aligned}
& \mathbf{v}_{\mathbf{2}_{\mathbf{n}}}=\overrightarrow{\mathbf{u}_{\mathbf{n}}} \cdot \overrightarrow{\mathbf{v}_{\mathbf{2}}} \\
& \mathbf{v}_{\mathbf{2}_{\mathrm{t}}}=\overrightarrow{\mathbf{u}_{\mathrm{t}}} \cdot \overrightarrow{\mathbf{v}_{\mathbf{2}}}
\end{aligned}
$$

Because tangential components do not change during the collision, then $\mathbf{v}_{\mathbf{1}_{\mathbf{t}}}^{\prime}=\mathbf{v}_{\mathbf{1}_{\mathbf{t}}}$ and $\mathbf{v}_{\mathbf{2}_{\mathbf{t}}}^{\prime}=\mathbf{v}_{\mathbf{2}_{\mathrm{t}}}$.

To get the normal components of the velocity vector after the collision, a formula similar to the $1 \mathrm{D}$ case could be used

$$
\mathbf{v}_{\mathbf{1}_{\mathrm{n}}}^{\prime}=\frac{\left(\mathbf{m}_{1}-\mathbf{m}_{2}\right) \mathbf{v}_{\mathbf{1}_{\mathbf{n}}}+2 \mathbf{m}_{2} \mathbf{v}_{\mathbf{2}_{\mathbf{n}}}}{\mathbf{m}_{1}+\mathbf{m}_{2}}
$$

and

$$
\mathbf{v}_{\mathbf{n}_{\mathbf{n}}}^{\prime}=\frac{\left(\mathbf{m}_{2}-\mathbf{m}_{1}\right) \mathbf{v}_{\mathbf{2}_{\mathbf{n}}}+2 \mathbf{m}_{1} \mathbf{v}_{\mathbf{1}_{\mathbf{n}}}}{\mathbf{m}_{1}+\mathbf{m}_{2}}
$$

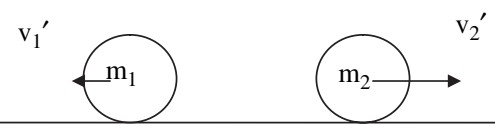

Fig. 2. The figure shows two particles of masses, $\mathbf{m}_{1}$ and $\mathbf{m}_{2}$, and velocities, $v_{1}^{\prime}$ and $v_{2}^{\prime}$, after they have collided (1-D collision).
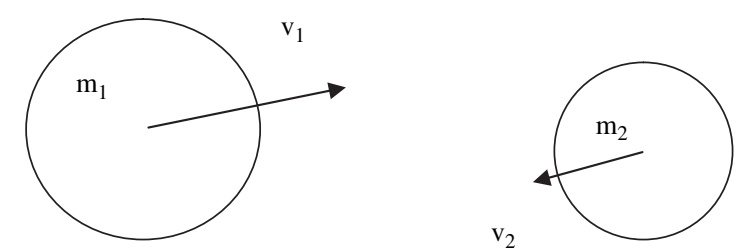

Fig. 3. The figure shows two particles of masses, $\mathbf{m}_{\mathbf{1}}$ and $\mathbf{m}_{\mathbf{2}}$, and velocities, $\mathbf{v}_{\mathbf{1}}$ and $\mathbf{v}_{\mathbf{2}}$, before they collide (2-D collision).

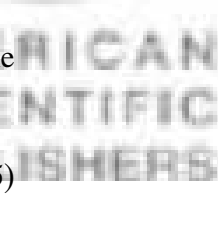
collision.

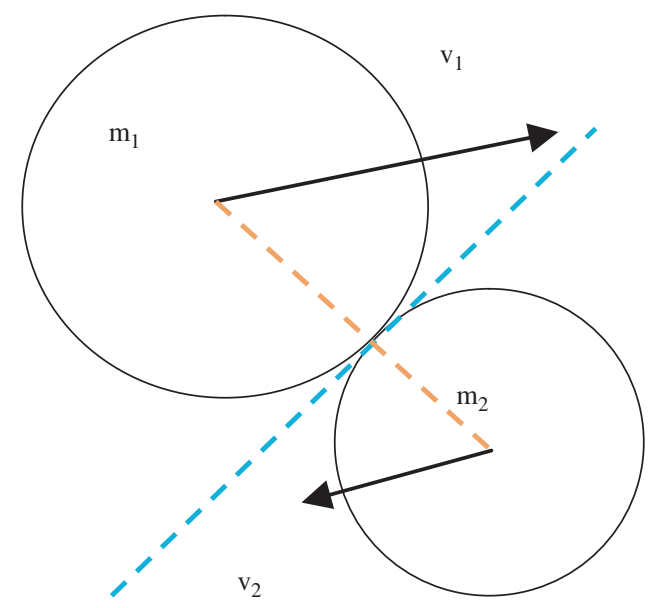

Fig. 4. The figure shows two particles of masses, $\mathbf{m}_{1}$ and $\mathbf{m}_{\mathbf{2}}$, and initial velocities, $\mathbf{v}_{\mathbf{1}}$ and $\mathbf{v}_{\mathbf{2}}$, at the moment of collision (2-D collision).

In Figure 6, to calculate the velocity vectors after the collision, normal vectors are multiplied by the corresponding scalar velocity components:

$$
\begin{aligned}
& \overrightarrow{\mathbf{v}_{\mathbf{1}_{\mathbf{n}}^{\prime}}^{\prime}}=\mathbf{v}_{\mathbf{1}_{\mathbf{n}}^{\prime}}^{\prime} \overrightarrow{\mathbf{u}_{\mathbf{n}}} \\
& \overrightarrow{\mathbf{v}_{\mathbf{1}_{\mathbf{t}}}^{\prime}}=\mathbf{v}_{\mathbf{1}_{\mathbf{t}}} \overrightarrow{\mathbf{u}_{\mathbf{t}}}
\end{aligned}
$$

and

$$
\begin{aligned}
& \overrightarrow{\mathbf{v}_{\mathbf{2}_{\mathbf{n}}}^{\prime}}=\mathbf{v}_{\mathbf{2}_{\mathbf{n}}}^{\prime} \overrightarrow{\mathbf{u}_{\mathbf{n}}} \\
& \overrightarrow{\mathbf{v}_{\mathbf{2}_{\mathbf{t}}}^{\prime}}=\mathbf{v}_{\mathbf{2}_{\mathbf{t}}} \overrightarrow{\mathbf{u}_{\mathbf{t}}}
\end{aligned}
$$

Finally, to get each object's velocity after the collision, normal and tangential vector components are added as shown in Eqs. (21) and (22).

$$
\begin{aligned}
& \overrightarrow{\mathbf{v}_{1}^{\prime}}=\overrightarrow{\mathbf{v}_{\mathbf{1}_{\mathbf{n}}}^{\prime}}+\overrightarrow{\mathbf{v}_{\mathbf{1}_{\mathbf{t}}}^{\prime}} \\
& \overrightarrow{\mathbf{v}_{\mathbf{2}}^{\prime}}=\overrightarrow{\mathbf{v}_{\mathbf{2}_{\mathbf{n}}}^{\prime}}+\overrightarrow{\mathbf{v}_{\mathbf{2}_{\mathbf{t}}}^{\prime}}
\end{aligned}
$$

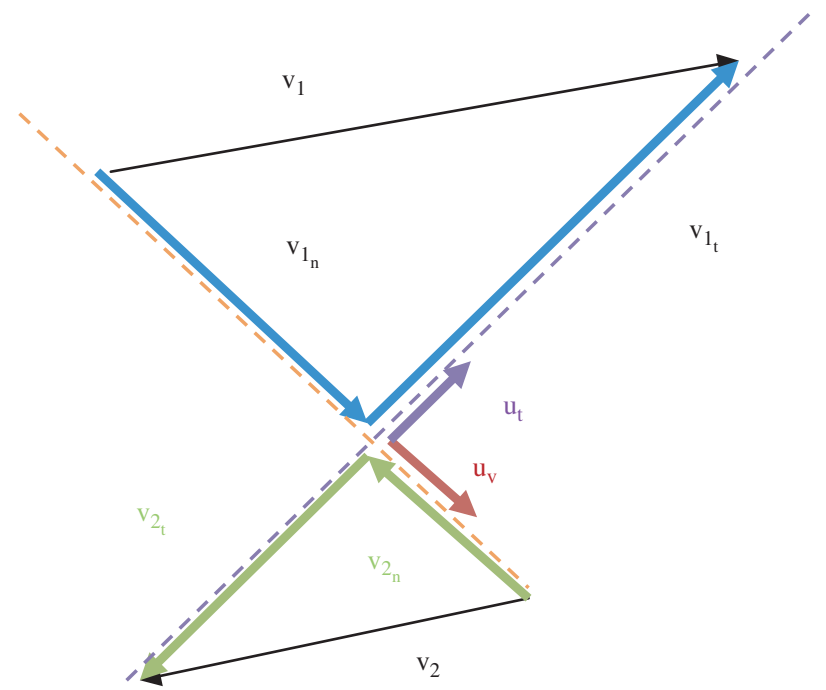

Fig. 5. The figure shows the vector components of the velocities before 


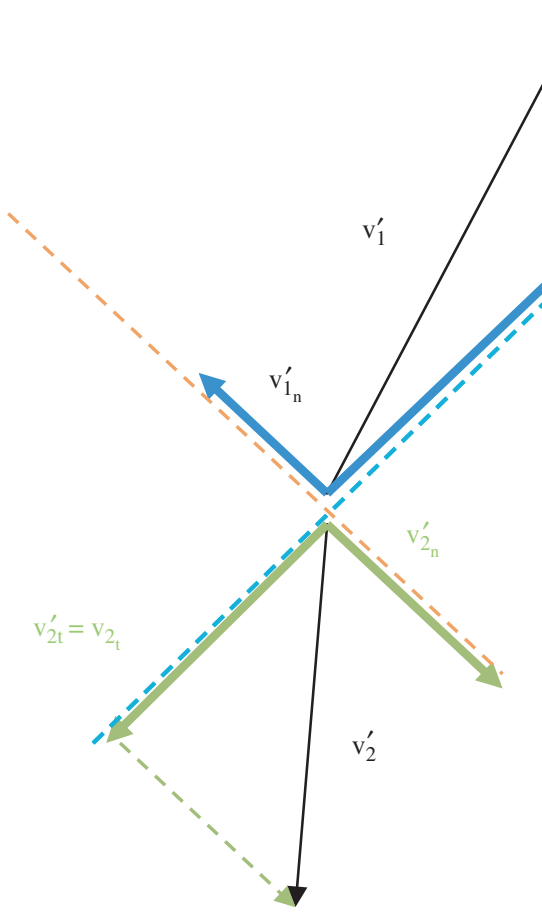

Fig. 6. The figure shows the vector components of the velocities after the collision.

\subsubsection{Collision in 3 Dimensions (3D)}

Similar to the 2D case, the normal unit vector of the collision was found, but instead of having one tangential component, there are 2 orthogonal components because now we have a plane rather than a line of collision.

To find the normal vector of the plane of collision:

$$
\overrightarrow{\mathbf{u}}_{\mathbf{n}}=\frac{\overrightarrow{\mathbf{n}}}{|\overrightarrow{\mathbf{n}}|}
$$

Where,

$$
\overrightarrow{\mathbf{n}}=\left(\left(\mathbf{x}_{2}-\mathbf{x}_{1}\right),\left(\mathbf{y}_{2}-\mathbf{y}_{1}\right),\left(\mathbf{z}_{2}-\mathbf{z}_{1}\right)\right)
$$

An example of a pair of tangential unit vectors is given below:

$$
\begin{aligned}
& \overrightarrow{\mathbf{t}_{\mathbf{1}}}=\left(\left(\mathbf{u}_{\mathbf{n}_{\mathbf{y}}}-\mathbf{u}_{\mathbf{n}_{\mathbf{z}}}\right),\left(\mathbf{u}_{\mathbf{n}_{\mathrm{z}}}-\mathbf{u}_{\mathbf{n}_{\mathbf{x}}}\right),\left(\mathbf{u}_{\mathbf{n}_{\mathbf{x}}}-\mathbf{u}_{\mathbf{n}_{\mathbf{y}}}\right)\right)
\end{aligned}
$$

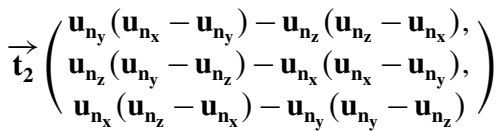

$\mathbf{u}_{\mathbf{n}_{\mathbf{x}}}, \mathbf{u}_{\mathbf{n}_{\mathbf{y}}}, \mathbf{u}_{\mathbf{n}_{\mathbf{z}}}$ are the $\mathbf{x}, \mathbf{y}$ and $\mathbf{z}$ coordinates of the collision normal vector, $\mathbf{t}_{\mathbf{1}}$ and $\mathbf{t}_{\mathbf{2}}$ are the tangential vectors. Their unit vectors with $\mathbf{x}, \mathbf{y}$ and $\mathbf{z}$ components are given below:

$$
\begin{aligned}
& \overrightarrow{\mathbf{u}}_{\mathbf{t}_{1}}=\frac{\overrightarrow{\mathbf{t}_{1}}}{\left|\overrightarrow{\mathbf{t}_{1}}\right|} \\
& \overrightarrow{\mathbf{u}}_{\mathrm{t}_{2}}=\frac{\overrightarrow{\mathbf{t}_{2}}}{\left|\overrightarrow{\mathbf{t}_{2}}\right|}
\end{aligned}
$$

Again, similar to the 2D case, we can calculate velocity components using the dot product. The normal component of $\mathbf{v}_{\mathbf{1}}$ velocity is:

$$
\mathbf{v}_{\mathbf{1}_{\mathbf{n}}}=\overrightarrow{\mathbf{u}_{\mathrm{n}}} \cdot \overrightarrow{\mathbf{v}_{1}}
$$

and $\mathbf{v}_{\mathbf{1}_{\mathbf{t}_{1}}}$ and $\mathbf{v}_{\mathbf{1}_{\mathbf{t}_{2}}}$ are its tangential components:

$$
\begin{aligned}
& \mathbf{v}_{\mathbf{1}_{\mathbf{t}_{1}}}=\overrightarrow{\mathbf{u}_{\mathbf{t}_{1}}} \cdot \overrightarrow{\mathbf{v}_{\mathbf{1}}} \\
& \mathbf{v}_{\mathbf{1}_{\mathbf{t}_{2}}}=\overrightarrow{\mathbf{u}_{\mathbf{t}_{2}}} \cdot \overrightarrow{\mathbf{v}_{\mathbf{1}}}
\end{aligned}
$$

To obtain the second object's velocity components, $\mathbf{v}_{\mathbf{2}_{\mathbf{n}}}, \mathbf{v}_{\mathbf{2}_{\mathbf{1}}}$, and $\mathbf{v}_{\mathbf{t}_{\mathbf{t}}}$, we use the relations below:

$$
\begin{aligned}
\mathbf{v}_{\mathbf{2}_{\mathbf{n}}} & =\overrightarrow{\mathbf{u}_{\mathbf{n}}} \cdot \overrightarrow{\mathbf{v}_{2}} \\
\mathbf{v}_{\mathbf{t}_{\mathbf{t}_{1}}} & =\overrightarrow{\mathbf{u}_{\mathbf{t}_{1}}} \cdot \overrightarrow{\mathbf{v}_{2}} \\
\mathbf{v}_{\mathbf{t}_{\mathbf{t}_{2}}} & =\overrightarrow{\mathbf{u}_{\mathrm{t}_{2}}} \cdot \overrightarrow{\mathbf{v}_{2}}
\end{aligned}
$$

Similar to the $2 \mathrm{D}$ case, the tangential components are equal before and after the collision. The normal velocity components are obtained similar to the 1D and 2D cases (Eqs. (15) and (16)).

$$
\begin{aligned}
\mathbf{v}_{\mathbf{1}_{\mathbf{n}}}^{\prime} & =\frac{\left(\mathbf{m}_{1}-\mathbf{m}_{2}\right) \mathbf{v}_{\mathbf{1}_{\mathbf{n}}}+2 \mathbf{m}_{2} \mathbf{v}_{\mathbf{2}_{\mathrm{n}}}}{\mathbf{m}_{1}+\mathbf{m}_{2}} \\
\mathbf{v}_{\mathbf{2}_{\mathrm{n}}}^{\prime} & =\frac{\left(\mathbf{m}_{2}-\mathbf{m}_{1}\right) \mathbf{v}_{2_{\mathrm{n}}}+2 \mathbf{m}_{1} \mathbf{v}_{\mathbf{1}_{\mathbf{n}}}}{\mathbf{m}_{1}+\mathbf{m}_{2}}
\end{aligned}
$$

Therefore the velocity vectors after collision become:

$$
\begin{aligned}
& \overrightarrow{v_{1}^{\prime}}=\overrightarrow{v_{\mathbf{1}_{n}}^{\prime}}+\overrightarrow{\mathbf{v}_{\mathbf{t}_{t_{1}}^{\prime}}^{\prime}}+\overrightarrow{\mathbf{v}_{\mathbf{t}_{2}}^{\prime}} \\
& \overrightarrow{\mathbf{v}_{2}^{\prime}}=\overrightarrow{\mathbf{v}_{\mathbf{2}_{\mathrm{n}}}^{\prime}}+\overrightarrow{\mathbf{v}_{\mathbf{t}_{1}}^{\prime}}+\overrightarrow{\mathbf{v}_{\mathbf{t}_{2}}^{\prime}}
\end{aligned}
$$

More simplification can be made to Eqs. (37 and 38). Note that $\mathbf{v}_{\mathbf{1}_{\mathbf{t}_{1}}}^{\prime}=\mathbf{v}_{\mathbf{1}_{\mathbf{t}_{1}}}, \mathbf{v}_{\mathbf{t}_{\mathbf{t}_{2}}}^{\prime}=\mathbf{v}_{\mathbf{1}_{\mathbf{t}_{2}}}$ and $\mathbf{v}_{\mathbf{t}_{\mathbf{t}_{1}}}^{\prime}=\mathbf{v}_{\mathbf{2}_{\mathbf{t}_{1}}}, \mathbf{v}_{\mathbf{t}_{\mathbf{t}_{2}}}^{\prime}=\mathbf{v}_{\mathbf{2}_{\mathbf{t}_{2}}}$, so

$$
\overrightarrow{\mathbf{v}_{1}}=\overrightarrow{\mathbf{v}_{\mathbf{n}_{\mathrm{n}}}}+\overrightarrow{\mathbf{v}_{\mathbf{t}_{1}}}+\overrightarrow{\mathbf{v}_{\mathbf{t}_{2}}}
$$

Furthermore,

$$
\overrightarrow{\mathbf{v}_{\mathbf{t}_{1}}}+\overrightarrow{\mathbf{v}_{\mathbf{t}_{2}}}=\overrightarrow{\mathbf{v}_{\mathbf{1}}}-\overrightarrow{\mathbf{v}_{\mathbf{1}_{\mathrm{n}}}}
$$

Equations (37) and (38) become:

$$
\begin{aligned}
& \overrightarrow{v_{1}^{\prime}}=\overrightarrow{v_{1_{n}}^{\prime}}+\overrightarrow{v_{1}}-\overrightarrow{v_{1_{n}}} \\
& \overrightarrow{v_{2}^{\prime}}=\overrightarrow{v_{\mathbf{2}_{\mathbf{n}}^{\prime}}^{\prime}}+\overrightarrow{\mathbf{v}_{\mathbf{2}}}-\overrightarrow{\mathbf{v}_{\mathbf{2}_{\mathbf{n}}}}
\end{aligned}
$$

respectively.

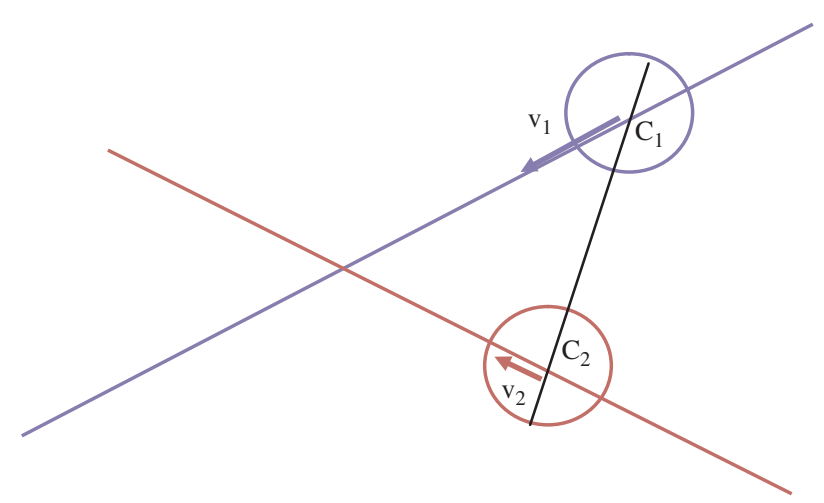

Fig. 7. The figure shows the schematic of two molecules before collision. 


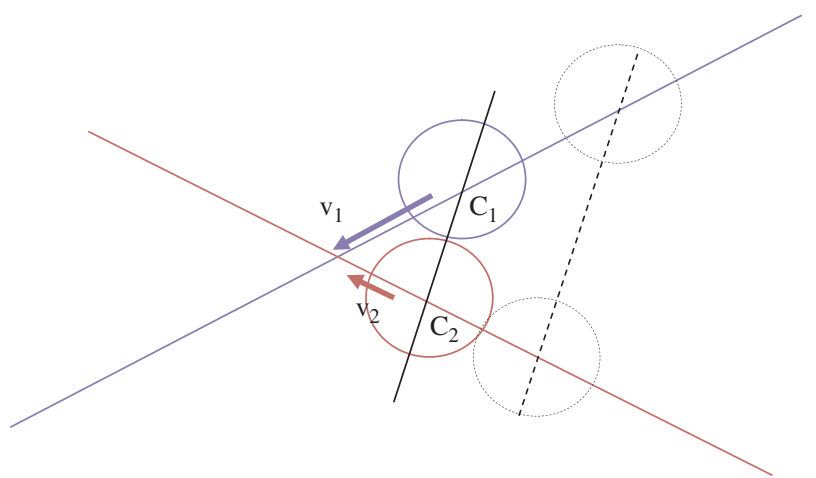

Fig. 8. The figure shows the schematic of two molecules at the moment of collision.

\subsection{Collision Detection}

If two objects are approaching each other as shown in Figure 7,

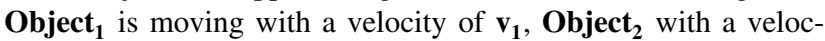
ity of $\mathbf{v}_{2} \cdot \mathbf{C}_{\mathbf{1}}$ and $\mathbf{C}_{\mathbf{2}}$ are the objects' centers of mass. The line segment connecting $\mathbf{C}_{\mathbf{1}}$ and $\mathbf{C}_{\mathbf{2}}$ should be parallel to itself until the objects collide. Because the model is based on discrete time intervals and not continuous ones, the exact moment of the collision cannot be caught or determined. Refer to Figure 8.

Consider that any two particles have collided when the distance between $\mathbf{C}_{\mathbf{1}}$ and $\mathbf{C}_{\mathbf{2}}$ is smaller than the sum of the two radii (refer to Fig. 9). The delta_t sampling time interval should be small enough to detect any collisions taking place. Since the line segment between $\mathbf{C}_{\mathbf{1}}$ and $\mathbf{C}_{\mathbf{2}}$ is always heading in the same direction, i.e., parallel to its previous state, it is not necessary to determine the exact moment of collision to get an approximation of the normal vector of the collision. The direction shown by the $\mathbf{C}_{\mathbf{1}}$ and $\mathbf{C}_{\mathbf{2}}$ line segment is:

$$
\overrightarrow{\mathbf{n}}=\left(\left(\mathbf{x}_{2}-\mathbf{x}_{1}\right),\left(\mathbf{y}_{2}-\mathbf{y}_{1}\right),\left(\mathbf{z}_{2}-\mathbf{z}_{1}\right)\right)
$$

\subsection{Flowchart}

MATLAB was used to simulate the movement of tRNA molecules in the bacterial cytoplasm. A flowchart was developed to organize the different subroutines. The computational input data, representing the initial conditions, are based on biological measurements and are summarized in Tables I and II. During initialization, we create a simulation space, place particles inside

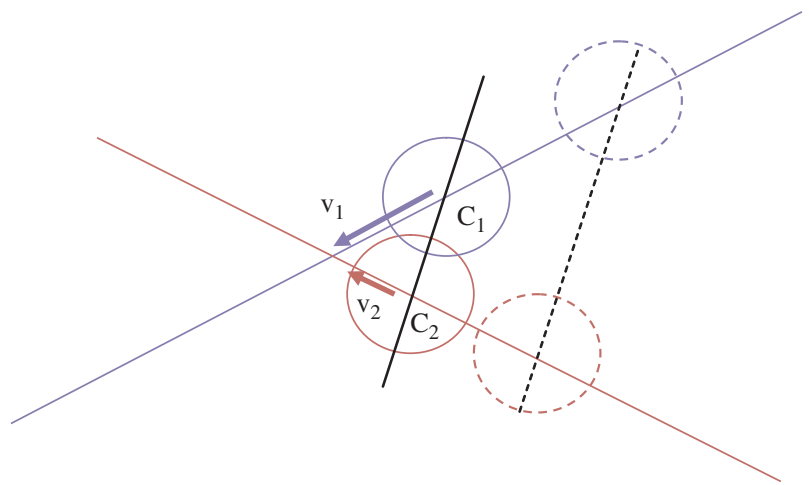

Fig. 9. The figure shows the schematic of two molecules virtually overlapping at the moment of collision.
Table I. Number of proteins, ribosomes, tRNAs, and other molecules in E. coli. ${ }^{5}$

\begin{tabular}{lc}
\hline Quantity & Molecule types \\
\hline 225,000 & Proteins \\
15,000 & Ribosomes \\
170,000 & tRNA-Molecules \\
$15,000,000$ & Small organic molecules \\
$25,000,000$ & lons \\
$70 \%$ & Water \\
\hline
\end{tabular}

that space, randomly assign each a mass, radius, initial velocity and location. Placing objects one by one into the simulation space guarantees that no object overlaps with another. Ribosome's velocities are assumed to be zero and simulated as fixed objects in the cytoplasm.

The simulation calculates new locations for each particle every delta_t time interval, checks whether any collision occurs or if any particle is located outside the simulation space. If a collision is detected, then the new velocities are calculated for both particles involved. Additionally, when any particle crosses the boundary of the simulation space, it would change direction. After each step, the program checks whether any cognate tRNA reached the target (the ribosome) located in the centre of our simulation space. Figure 10 shows the flowchart used to code the MATLAB program.

To calculate the velocity of particles after collision, the following equations from the previous section were used in the MATLAB code: Collision normal vector:

$$
\overrightarrow{\mathbf{n}}=\left(\left(\mathbf{x}_{\mathbf{2}}-\mathbf{x}_{1}\right),\left(\mathbf{y}_{2}-\mathbf{y}_{1}\right),\left(\mathbf{z}_{2}-\mathbf{z}_{1}\right)\right)
$$

Normal unit vector:

$$
\overrightarrow{\mathbf{u}}_{\mathbf{n}}=\frac{\overrightarrow{\mathbf{n}}}{|\overrightarrow{\mathbf{n}}|}
$$

Table II. E. coli cell dimensions. ${ }^{6}$

Cell length

Cell diameter

Cell total volume

Average size of protein

Average diameter of ave. protein

Average MW of protein

Average size of mRNA

Average length of mRNA

Mean Velocity of $70 \mathrm{kD}$ protein (cytoplasm)

Mean Velocity of $40 \mathrm{kD}$ protein (cytoplasm)

Mean Velocity of $30 \mathrm{kD}$ protein (cytoplasm)

Mean Velocity of $14 \mathrm{kD}$ protein (cytoplasm)

Mean Velocity of small molecules (cytoplasm)

Volume occupied by water

Volume occupied by protein

Volume occupied by all RNA

Volume occupied by rRNA

Volume occupied by tRNA

Volume occupied by mRNA

Volume occupied by DNA

Volume occupied by ribosomes

Translation rate

Number of mRNA/cell

Number of tRNA/cell

Number of ribosomes/cell

MW of ribosome

Diameter of ribosome

Volume of ribosome
$2 \mu \mathrm{m}$ or $2 \times 10^{-6} \mathrm{~m}$ $0.8 \mu \mathrm{m}$ or $0.8 \times 10^{-6} \mathrm{~m}$ $1 \times 10^{-15} \mathrm{~L}$ or $1 \times 10^{-18} \mathrm{~m}^{3}$ (other est. at $0.88 \times 10^{-15} \mathrm{~L}$ ) 360 residues $5 \mathrm{~nm}$ $40 \mathrm{kD}$ 1100 bases $370 \mathrm{~nm}$

$3 \mathrm{~nm} / \mathrm{ms}=3 \times 10^{-6} \mathrm{~m} / \mathrm{s}$ $5 \mathrm{~nm} / \mathrm{ms}=5 \times 10^{-6} \mathrm{~m} / \mathrm{s}$

$7 \mathrm{~nm} / \mathrm{ms}=7 \times 10^{-6} \mathrm{~m} / \mathrm{s}$

$10 \mathrm{~nm} / \mathrm{ms}=10 \times 10^{-6} \mathrm{~m} / \mathrm{s}$ $50 \mathrm{~nm} / \mathrm{ms}=5 \times 10^{-5} \mathrm{~m} / \mathrm{s}$ $70 \%$ $17 \%$ $6 \%$ $5 \%$ $0.8 \%$ $0.2 \%$ $1 \%$ $8 \%$ $40 \mathrm{aa} / \mathrm{sec}$ 4000

200,000

18,000

$2700 \mathrm{kD}$

$20 \mathrm{~nm}$ $4.2 \times 10^{-24} \mathrm{~m}^{3}$ 


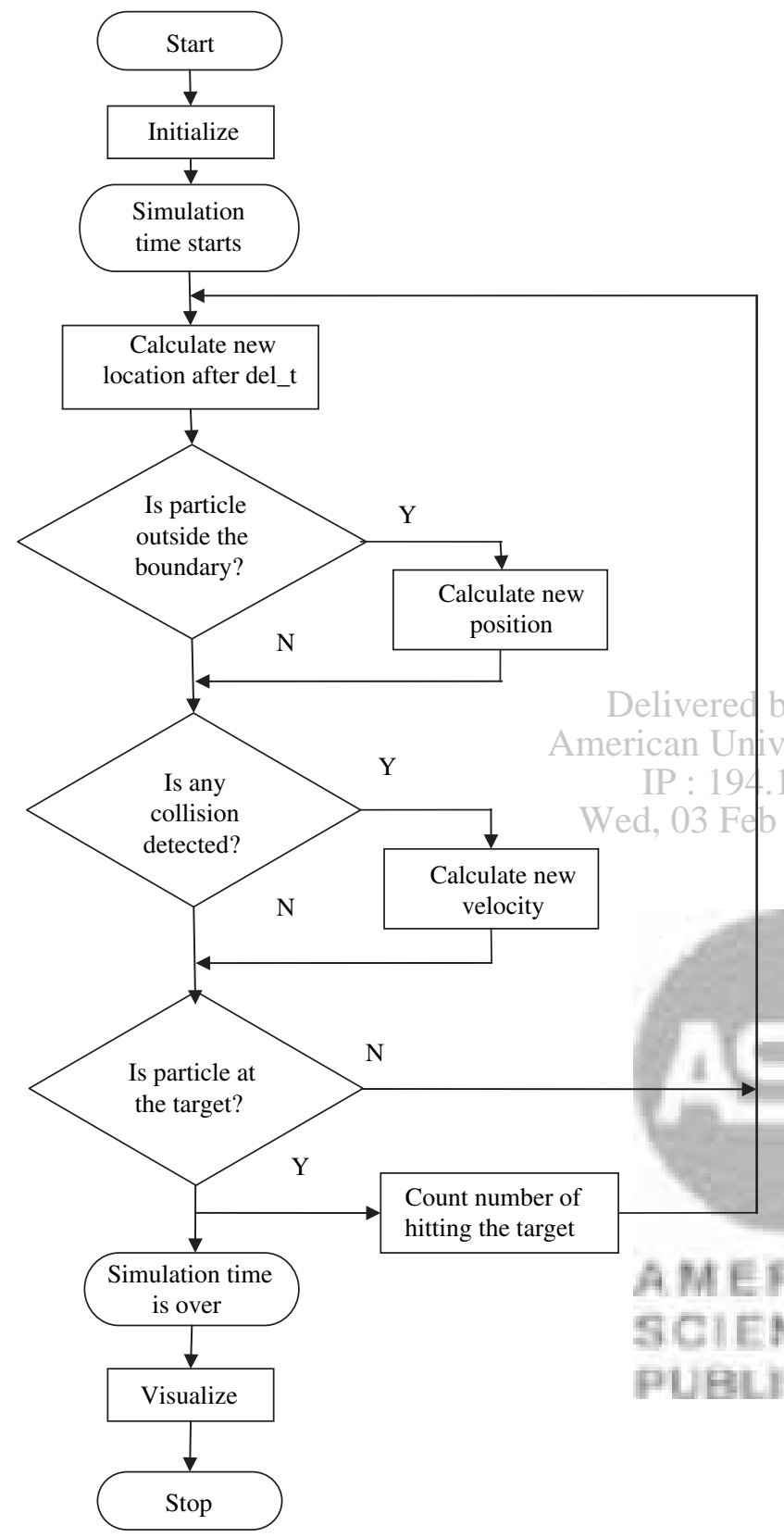

Fig. 10. The figure shows the Flowchart of the MATLAB algorithm used to simulate ctRNA/ribosomal interactions.

Object $_{1}$ velocity normal component before collision:

$$
\mathbf{v}_{\mathbf{1}_{\mathrm{n}}}=\overrightarrow{\mathbf{u}_{\mathrm{n}}} \cdot \overrightarrow{\mathbf{v}_{1}}
$$

Object $_{2}$ velocity normal component before collision:

$$
\mathbf{v}_{\mathbf{2}_{\mathbf{n}}}=\overrightarrow{\mathbf{u}_{\mathbf{n}}} \cdot \overrightarrow{\mathbf{v}_{2}}
$$

The velocity values after the collision are:

Object $_{1}$ normal component:

$$
\begin{gathered}
\mathbf{v}_{\mathbf{1}_{\mathbf{n}}}^{\prime}=\frac{\left(\mathbf{m}_{1}-\mathbf{m}_{2}\right) \mathbf{v}_{\mathbf{1}_{\mathbf{n}}}+2 \mathbf{m}_{2} \mathbf{v}_{\mathbf{2}_{\mathbf{n}}}}{\mathbf{m}_{1}+\mathbf{m}_{2}} \\
\overrightarrow{\mathbf{v}_{\mathbf{1}_{\mathrm{n}}}^{\prime}}=\mathbf{v}_{\mathbf{1}_{\mathbf{n}}}^{\prime} \overrightarrow{\mathbf{u}_{\mathbf{n}}}
\end{gathered}
$$

Object $_{2}$ normal component:

$$
\begin{gathered}
\mathbf{v}_{2_{\mathbf{n}}}^{\prime}=\frac{\left(\mathbf{m}_{2}-\mathbf{m}_{1}\right) \mathbf{v}_{2_{\mathbf{n}}}+2 \mathbf{m}_{1} \mathbf{v}_{\mathbf{1}_{\mathbf{n}}}}{\mathbf{m}_{1}+\mathbf{m}_{2}} \\
\overrightarrow{\mathbf{v}_{2_{\mathbf{n}}}^{\prime}}=\mathbf{v}_{\mathbf{2}_{\mathbf{n}}}^{\prime} \overrightarrow{\mathbf{u}_{\mathbf{n}}}
\end{gathered}
$$

Object $_{1}$ velocity:

$$
\overrightarrow{\mathbf{v}_{1}^{\prime}}=\overrightarrow{\mathbf{v}_{\mathbf{1}_{\mathbf{n}}}^{\prime}}+\overrightarrow{\mathbf{v}_{\mathbf{1}}}-\overrightarrow{\mathbf{v}_{\mathbf{1}_{\mathbf{n}}}}
$$

Object $_{2}$ velocity:

$$
\overrightarrow{\mathbf{v}_{2}^{\prime}}=\overrightarrow{\mathbf{v}_{\mathbf{2}_{\mathbf{n}}}^{\prime}}+\overrightarrow{\mathbf{v}_{2}}-\overrightarrow{\mathbf{v}_{\mathbf{n}_{\mathbf{n}}}}
$$

\section{SIMULATION}

After developing the computer program to simulate the tRNA movement, simulations were run using the settings shown in Table III. to

For visualization purposes an 'avi' movie file was created using the particles' location matrix. Each frame comprises the 3D MATLAB graph taken every delta_t time interval. In Figure 11, we can see the central ribosome with the mRNA and ctRNAs moving in simulation space. For simplicity, objects other than ctRNA are not shown; but are involved in the simulation, including other ribosomes (Fig. 12) and other noncognate tRNAs (Fig. 13), different proteins as well as other macromolecules capable of diverting the path of tRNAs in the cytoplasm. Figure 14 shows the simulation with 1,000 other objects present, it fills about $5 \%$ of the simulation space while Figure 15 shows the presence of 10,000 other particles in addition to 144 ribosomes, and more than 1600 tRNAs. In the latter figure,

\begin{tabular}{|c|c|c|}
\hline \multicolumn{2}{|r|}{ Simulation sets } & \multirow{2}{*}{$\begin{array}{c}\text { Results } \\
\text { (Average of } 10 \text { runs) }\end{array}$} \\
\hline Serial number & Special conditions & \\
\hline 1 & Normal cell & 0.3 \\
\hline 2 & Cell is dry ${ }^{1}$ & 0.1 \\
\hline 3 & Diluted cytoplasm² & 0.3 \\
\hline 4 & Dry cell $+5 \times$ longer time frame ${ }^{3}$ & 0.9 \\
\hline 5 & ctRNA density doubled ${ }^{4}$ & 0.2 \\
\hline 6 & ctRNA density tripled ${ }^{5}$ & 0.4 \\
\hline 7 & Velocity doubled ${ }^{6}$ & 0.2 \\
\hline 8 & ctRNAs velocity agitated $(\times 10)^{7}$ & 2 \\
\hline 9 & Sampling rate increased $(\times 2)^{8}$ & 0.1 \\
\hline
\end{tabular}
these molecules occupy $32 \%$ of the simulation space.

The model was simulated in a $200 \mathrm{~nm} \times 200 \mathrm{~nm} \times 200 \mathrm{~nm}$ cube. The particles are spaced randomly throughout the cube. The ribosome radius is set to $10 \mathrm{~nm}$, tRNA radius to $3.5 \mathrm{~nm}$, and the size of other macromolecules, capable of affecting the movement of tRNA under investigation, are randomly selected to be

Table III. Results of the simulation.

${ }^{1}$ Simulated by doubling the number of other particles in the cytoplasm.

${ }^{2}$ Number of other particles in the cytoplasm is reduced by $80 \%$.

${ }^{3}$ Initial conditions are selected similar to set \#2, but simulation time is increased by $400 \%$, (simulating slower metabolism).

${ }^{4}$ Number of ctRNAs are increased.

${ }^{5}$ The initial velocity of all moving objects in the simulation space is doubled

${ }^{6}$ The initial velocity of all moving objects in the simulation space is tripled.

${ }^{7}$ Only ctRNAs initial velocity is increased 10 fold, to simulate a case when ctRNAs are able to move faster than other noncognate tRNAs.

${ }^{8}$ These simulations are meant to check whether our estimation for the sampling rate is correct and no collisions are overlooked. 


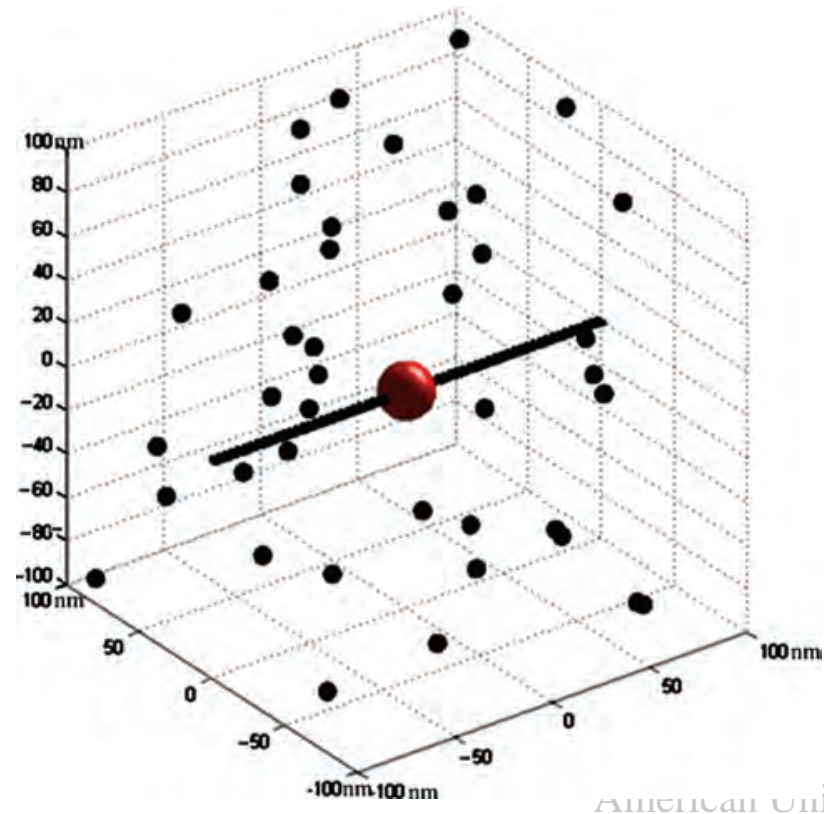

Fig. 11. The figure shows the MATLAB simulation space.

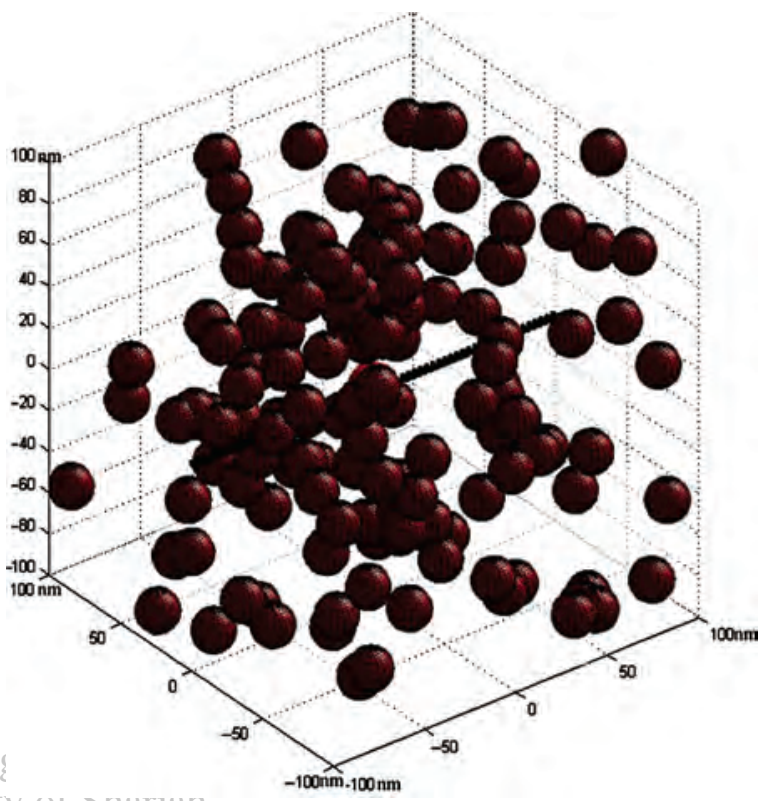

Fig. 13. 2The figure shows the simulation space with 1600 tRNAs. between 5 and $10 \mathrm{~nm}$ in radius. ${ }^{6}$ Initial ribosomal velocities are assumed to be zero, because these structures could be considered stationary in the cytoplasm. The initial velocities of the tRNA molecules and other particles are assumed to be $(10 \mathrm{~nm} / \mathrm{ms}){ }^{6}$ It is important to note here that all initial velocities are assumed to be omnidirectional.

\subsection{Results}

To have a basis for comparison, initially $40 \mathrm{ctRNAs}$ and the central ribosome (with the mRNA) are placed in the model space and 10 simulations are run. Table III summarizes the results.
The following particles are placed in the simulation space to simulate conditions in an average bacterial cytoplasm:

- Number of tRNA $=1600$

- Number of ctRNA $=40$

- Number of ribosomes $=144$

- Number of other particles $=5000$

The length of the simulation time is set to $20 \mathrm{~ms}$ which is the normal amino acid assembling rate. The simulation time step is selected to be $0.1 \mathrm{~ms}$ in order to detect all possible collisions among these particles. Each set of conditions was simulated 10 times. Table III summarizes the results of the average number of hits on the ribosome by the ctRNA. First, we simulate the average hits in a normal cell (i.e., a cell that has the

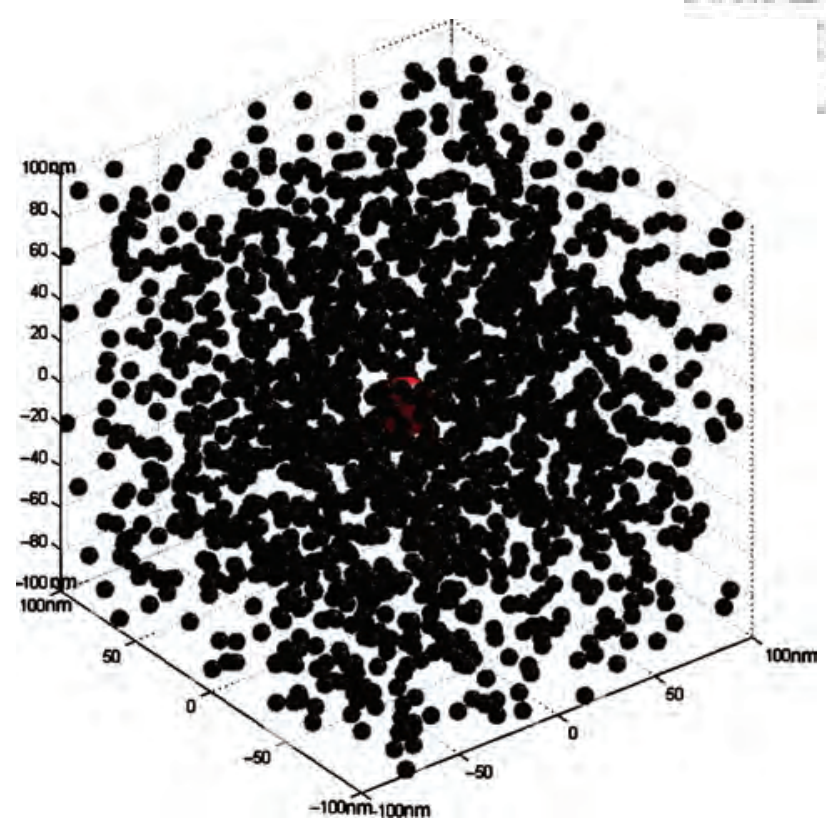

Fig. 12. The figure shows the simulation space with the messenger RNA and 144 ribosomes.

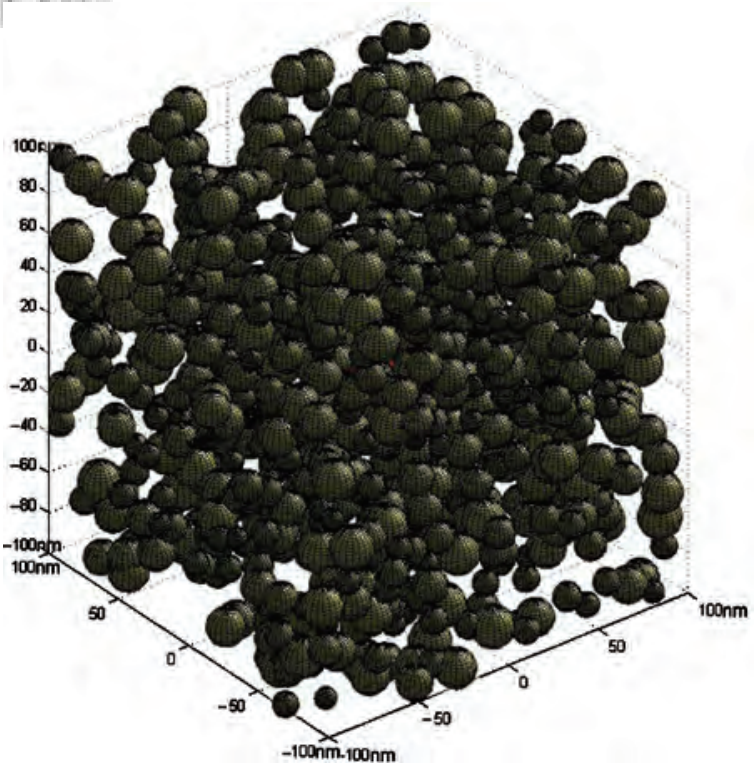

Fig. 14. The figure shows the sample space with 1,000 "other particles." 


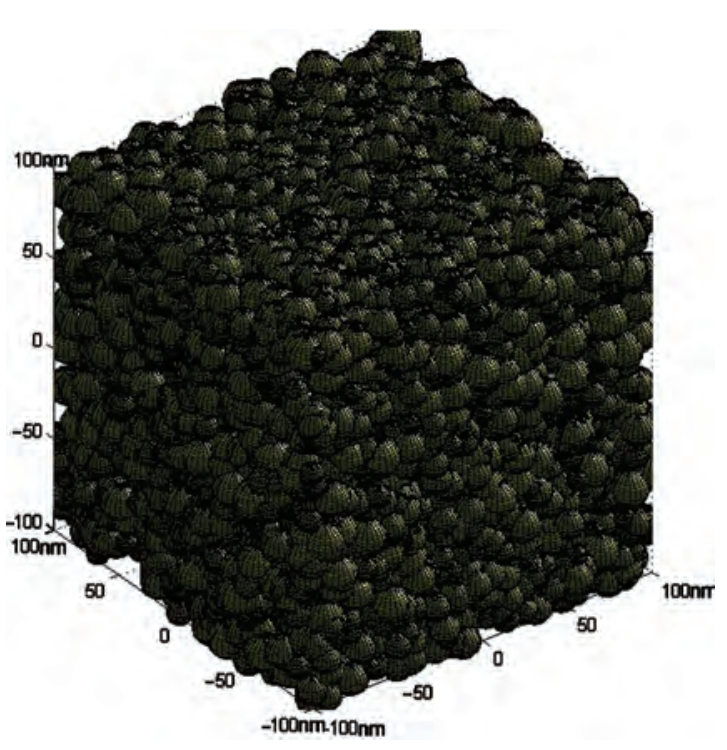

Fig. 15. The figure shows the sample space with 10,000 "other particles."

American Unive same number of tRNA, ctRNA, ribosomes and other large macromolecules as reported in literature for E. coli). Our results show that, on average, 0.3 ctRNAs reach the ribsome's surface. Thus, the probability of the ctRNA biologically binding to the actual Aminoacyl-tRNA binding site is much smaller. These findings lead us to reject the hypothesis that the ctRNA binding to the ribosome "A site" is a random process in E. coli. Next and in attempt to understand the mechanism involved in tRNA movement in the cytoplasm, we used different cellular conditions to simulate our dynamic system. We doubled the number of other particles in our simulation space. The results of 10 simulations show that the average ctRNAs reaching the surface decreased to 0.1 because these "other macromolecules" act as obstacles in the path of the ctRNA.

We also reduced the number of other particles by $80 \%$ and observed that on average $0.3 \mathrm{ctRNAs}$ reach the ribosome site. The average here is similar to that observed in a normal cell, but we presume that a slight difference could be observed between the two cases if more simulations were undergone. Next, we increased the length of simulation to $100 \mathrm{~ms}$ (instead of $20 \mathrm{~ms}$ ) to simulate slower metabolism. The results show that, on average, 0.9 ctRNA molecules hits the surface of the ribosome. Therefore, even by reducing the rate of amino acid assembly from the normal $50 \mathrm{aa} / \mathrm{second}$ to $10 \mathrm{aa} /$ second, which would give the ctRNA a better chance to reach the ribosome, less than 1 ctRNA is able to reach any location on the ribosomal surface.

We also used our code to study the effect of doubling and tripling the number of ctRNAs in our simulation space. Results show that even by significantly increasing the number of ctRNAs in the vicinity of the ribosome, the average hits recorded did not reach 1 . Table III shows that the only simulation that succeeded in increasing the number of hits on the ribosome was achieved when the speed of the ctRNA was increased 10 fold (the speed of all other molecules remained the same as used in our previous simulations). Our simulations averaged 2 ctRNA hits per run. It is important to reiterate that results shown in Table III report the average number of ctRNA reaching the surface of the ribosome in a given timeframe, it does not mean that the correct ctRNA is inserted in the "A site" of the ribosome, keeping in mind that the surface area of the ribosome is at least an order of magnitude larger than the "A site" itself. If we approximate the surface area of the ribosome as that of a sphere with a diameter of $20 \mathrm{~nm}$, we get the total ribosomal surface area of $1256 \mathrm{~nm}^{2}$. On the other hand, the area of the "A site" could be approximated as that of a circle similar in size to the tRNA cross-section. In this case, we get an "A site" area of $38.5 \mathrm{~nm}^{2}$. Thus, the ratio of the two areas is approximately 1:33, which means that any ctRNA that reaches the ribosome has a much smaller chance of binding to the ribosomal "A site".

\section{CONCLUSION}

Therefore it is obvious, that a prospective consecutive ctRNA molecule has no realistic chance of reaching a ribosome at a realistic rate in normal circumstances. The simulation shows that more than one ctRNA can hit the target-the ribosomal surface-only when the number of ctRNA-s or the time interval is exaggerated.

In Since the results of the simulations presented in this article proved that it is virtually impossible for the tRNA to reach the "A site" of the ribosome by random motion, other possible explanations for this biological phenomenon are given below. The ribosome could, although unlikely, store different types of tRNAs and preselect the cognate before entering the "A site." Another hypothesis is the existence of a signaling mechanism between the ribosome and the tRNA which allows for the recognition of the cognate and accelerates its movement towards the "A site." A third hypothesis is that tRNAs reach the ribosome in an orderly preselected manner whereby the messenger RNA (mRNA) is capable of filtering out the consecutive tRNAs from the cytoplasm, allowing the correct ctRNA to reach the "A site" in the ribosome. We will discuss these three hypotheses in the next article.

\section{ABBREVIATIONS}

Abbreviation

Aa

DNA

RNA

tRNA

ctRNA

mRNA

rRNA

$\mathrm{Nt}$

$\mathrm{Bp}$

Ms

E. coli

A site

\section{Term}

Aminoacyl

deoxyribonucleic acid

ribonucleic acid

transfer RNA

cognate tRNA

messenger RNA

ribosomal RNA

Nucleotide

base pair

Millisecond

Escherichia coli

aminoacyl-tRNA binding site
Acknowledgments: The authors would like to acknowledge the help of Dr. Erika Zilahi in this project.

\section{References and Notes}

1. G. M. Cooper, The Cell (1997), Chap. 7, p. 273.

2. H. Lodish, A. Berk, S. L. Zipursky, P. Matsudaira, D. Baltimore, and J. E. Danell, Molecular Cell Biology (2000), Chap. 4, p. 100.

3. K. Y. Sanbonmatsu, Biochimie 88, 1075 (2006).

4. C. S. Fraser and J. W. Hershey, Journal of Biology 4, 8 (2005). 
5. M. Schwehm, Parallel stochastic simulation of whole-cell models. Proceedings of the 2nd International Conference on Systems Biology, Los Angeles (2001).

6. Project CyberCell, Institute for Biomolecular Design. E. coli Statistics; [Online] http://redpoll.pharmacy.ualberta.ca/CCDB/cgi-bin/STAT NEW.cgi.

7. M. B. Elowitz, M. G. Surette, P. E. Wolf, J. B. Stock, and S. Liebler, Journal of Bacteriology 181, 197 (1999).

8. J. Deich, E. M. Judd, H. H. McAdams, and W. E. Moerner, PNAS 101, 15921 (2004)
9. I. Golding and E. C. Cox, Phys. Rev. Lett. 96 (2006).

10. G. Broderick, M. Ruaini, E. Chan, and M. J. Ellison, In Silico Biology 5, 163 (2005).

11. S. J. Plimpton and A. Slepoy, Journal of Physics 16, 305 (2005)

12. K. Y. Sanbonmatsu, S. Joseph, and C. S. Tung, PNAS 102, 15854 (2005).

13. K. Y. Sanbonmatsu, Biochimie 88, 1053 (2006).

14. Ch. Berchek, http://www.geocities.com/vobarian/2dcollisions/ (2006).

Received: 7 February 2009. Accepted: 7 May 2009.

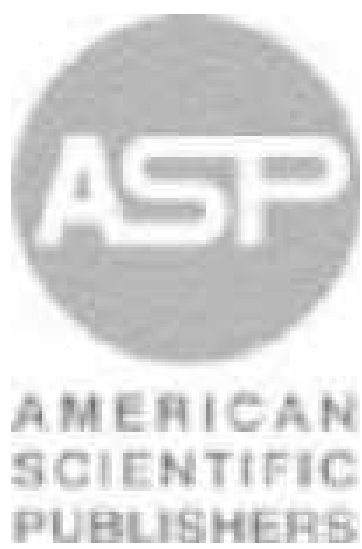

